\title{
Akutbehandlung des Schlaganfalls
}

\section{Die Zukunft gehört der mechanischen Rekanalisation}

\author{
Die einzige generell verfügbare kau- \\ sale Therapie beim akuten ischä- \\ mischen Insult ist die systemische Lyse. \\ Nach neueren Studienergebnissen \\ wurde das Zeitfenster auf 4,5 Stunden \\ erweitert. Ein weiterer Therapiefort- \\ schritt dürfte jedoch nur durch den \\ frühzeitigen Einsatz mechanischer \\ Rekanalisationsverfahren zu erreichen \\ sein.
}

"Auch wenn es sich beim Herzinfarkt und Schlaganfall nur um die unterschiedliche Lokalisation ein und derselben Erkrankung, nämlich der Atherothrombose handelt, so bestehen doch bei der Pathogenese, der Klinik und den Therapiemöglichkeiten wesentliche Unterschiede", sagte Prof. H. Poppert, München.

\section{Vielgestaltige Pathogenese}

Während es sich beim akuten Koronarsyndrom immer um eine Plaqueruptur mit Thrombusbildung und nachfolgender Ischämie handele, so sei die Pathogenese beim Schlaganfall vielgestaltig. In ca. $15 \%$ liegt eine intrazerebrale Blutung vor, und bei den Hirninfarkten werden zwei Drittel durch ein embolisches Geschehen und nur ein Drittel durch einen lokalen thrombotischen Verschluss wie beim Herzinfarkt verursacht., Die embolischen Hirninfarkte können entweder durch einen kardialen Thrombus bei Vorhof- flimmern oder durch arterio-arterielle Embolien hervorgerufen werden", so Poppert. Dabei handele es sich meist um große Hirninfarkte, während ein lokaler Verschluss eines kleinen Gefäßes meist nur zu einem kleinen lakunären Hirninfarkt führe. Darüber hinaus hat eine lyseinduzierte Blutung in das Zielorgan beim Herzinfarkt keine klinische Relevanz, beim Schlaganfall dagegen stellt sie nicht selten eine fatale Komplikation dar.

\section{3-Stunden-Fenster gekippt}

Die einzige bisher allgemein verfügbare kausale Therapieoption beim ischämischen Insult ist die intravenöse Lyse mit Alteplase. Früher galt ein Zeitfenster von drei Stunden. „Nach den Ergebnissen der ECASS-III-Studie sollte das Zeitfenster für den Beginn einer solchen Therapie heute auf 4,5 Stunden verbreitert werden", so Poppert. Doch diese Therapieempfehlung habe bisher kaum Akzeptanz gefunden. Nach aktuellen Studienergebnissen profitierten auch Patienten mit kleineren Insulten und auch betagte $\mathrm{Pa}$ tienten über 80 Jahren von einer solchen Therapie.

\section{Bei großen Kalibern ist die Erfolgsrate relativ gering}

Doch die intravenöse Lyse ist mit einer Reihe von Limitationen und Komplikationen assoziiert. So ist bei großen Gefäßkalibern die Erfolgsrate relativ gering und embolisches

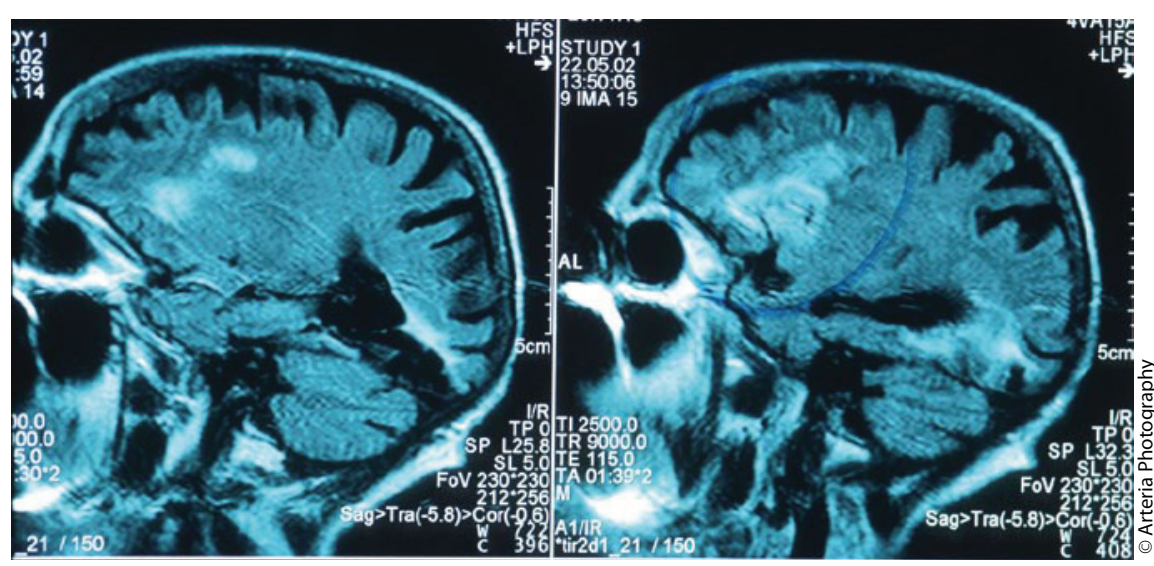

Schlaganfall: Die frühzeitige Therapie ist entscheidend für die Prognose.
Material lässt sich oft nicht lysieren. Auch kann die kurze Halbwertzeit des Thrombolytikums zu einem Reboundphänomen führen, d.h. nach initialer Besserung kommt es rasch zu einer Verschlechterung der klinischen Situation. Des Weiteren besteht ein Blutungsrisiko und es müssen Kontraindikationen beachtet werden.

\section{Mechanische Thrombektomie:} Ist das Outcome wirklich besser?

Eine entsprechende Metaanalyse zeigt, dass bei Einsatz der intravenösen Lyse die Rekanalisationsrate bei ca. $46 \%$ liegt. Durch die intraarterielle Gabe des Thrombolytikums kann sie auf $63 \%$ und durch den Einsatz mechanischer Rekanalisationsverfahren sogar auf $84 \%$ gesteigert werden., Und bei Einsatz dieses effektiveren Rekanalisationsverfahrens ist die Einblutungsgefahr nicht erhöht", so Poppert. Zurzeit würden eine Reihe von verschiedenen Devices für die mechanische Thrombektomie entwickelt und klinisch geprüft wie z. B. Aspirationskatheter und retrahierbare Stents.

Erste Erfahrungen mit diesen Systemen seien durchaus vielversprechend, obwohl nicht bei all diesen Verfahren trotz höherer Rekanalisationsrate ein besseres klinisches Outcome habe dokumentiert werden können. Um den Erfolg einer solchen Therapie besser beurteilen zu können, empfehle es sich, bei der Beurteilung der Rekanalisation nicht die in der Kardiologie etablierte TIMIKlassifikation anzuwenden, sondern die für die Behandlung des Schlaganfalls modifizierte TICI-Klassifikation.

„Entscheidend für die Prognose der Schlaganfallpatienten ist heute eine gute Infrastruktur, damit Patienten möglichst frühzeitig einer innovativen Therapie zugeführt werden können", so Poppert. Für die Zukunft seien mechanische Rekanalisationssysteme das vielversprechendste Therapiekonzept.

Dr. med. Peter Stiefelhagen II

I| Quelle: Seminarkongress „ Interdisziplinäre Intensivmedizin", 10.3.2011 in Garmisch-Partenkirchen. 